\title{
ARTROSCOPIA DIAGNÓSTICA E TERAPÊUTICA EM PACIENTES SINTOMÁTICOS PÓS-ARTROPLASTIA DO JOELHO
}

\author{
DIAGNOSTIC AND THERAPEUTIC ARTHROSCOPY IN SYMPTOMATIC \\ PATIENTS AFTER KNEE ARTHROPLASTY
}

Fabricio Roberto Severino', Clodoaldo José Duarte de Souza', Nilson Roberto Severino²

\section{RESUMO}

Objetivos: Verificar o valor da artroscopia na investigação e no tratamento da dor no joelho pós-artroplastia não esclarecida por exames clínicos e subsidiários. Métodos: Entre 402 pacientes submetidos à artroplastia total ou unicompartimental de setembro de 2001 a abril de 2007 num hospital universitário público, 17 deles apresentavam dor na articulação protética, sem diagnóstico clínico, radiográfico, laboratorial, cintilográfico ou por ressonância nuclear magnética. Todos foram submetidos à artroscopia e a sintomatologia foi avaliada pela escala de Lysholm, comparando-se os períodos pré e pós-artroscopia. Os achados intraoperatórios foram registrados. Resultados: O procedimento foi eficaz para alívio do sintoma doloroso em 14 dos 17 pacientes $(82,35 \%)$. A mediana do escore de Lysholm subiu de 36 pontos antes da artroscopia para 94 pontos após $(\mathrm{p}<0,001)$. A maioria dos pacientes (12) apresentava como diagnóstico artroscópico um quadro de fibrose conhecido como "ciclope"; nos outros cinco pacientes foi verificada sinovite anterior. Todos foram tratados com ressecção. Conclusões: A artroscopia do joelho pós-artroplastia em pacientes com dor persistente a diagnosticar revela quadros de artrofibrose localizada ("ciclopes") ou de sinovite, que podem ser tratados pelo mesmo procedimento, resultando em melhora do quadro álgico.

Descritores - Artroplastia do joelho; Artroscopia; Fibrose; Sinovite; Artralgia

\section{ABSTRACT}

Objectives: Assess the worthiness of arthroscopy in investigating and treating knee pain after arthroplasty unexplained by clinical and subsidiary examinations. Methods: Among 402 patients submitted to total or unicompartimental arthroplasty between September 2001 and April 2007 at a public university hospital, 17 presented with pain on prosthetic articulation, without clear diagnosis by clinical, X-ray, laboratory, scintiscan, or nuclear magnetic resonance tests. All patients were submitted to arthroscopy and symptoms were assessed by using the Lysholm scale, comparing pre- and post-arthroscopy periods. Peroperative findings have been recorded. Results: The procedure was effective for pain relief in 14 of 17 patients (82.35\%). The median for Lysholm scale climbed from 36 points before arthroscopy to 94 points after the procedure $(p<0.001)$. Most of the patients (12) were arthroscopically diagnosed with fibrosis known as "cyclop"; on the remaining five patients, anterior synovitis was found. All patients were treated by resection. Conclusions: Knee arthroscopy after arthroplasty in patients presenting unclear persistent pain shows localized arthrofibrosis ("cyclops") or synovitis, which can be treated by using the same procedure, resulting in pain relief.

Keywords - Knee arthroplasty; Arthroscopy; Fibrosis; Synovitis; Arthralgia

\footnotetext{
1 - Residente do Departamento de Ortopedia e Traumatologia, Faculdade de Ciências Médicas, Santa Casa de Misericórdia de São Paulo, São Paulo, Brasil.

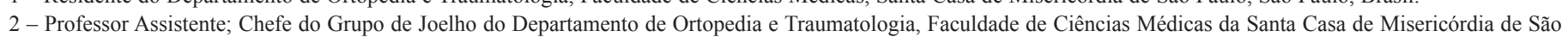
Paulo, São Paulo, Brasil.
}

Trabalho realizado no Departamento de Ortopedia e Traumatologia da Faculdade de Ciências Médicas da Santa Casa de Misericórdia de São Paulo. Correspondência: Nilson Roberto Severino, Rua Dias Leme, 134, apt ${ }^{\circ} 183$-03118-040. E-mail: nrseverino@uol.com.br 


\section{INTRODUÇÃO}

Um grande número de pacientes tem realizado procedimentos de artroplastia no joelho nas últimas décadas. Os resultados têm sido favoráveis na melhora do quadro de dor e na mobilidade da articulação, limitada antes do procedimento como consequência de osteoartrose, artrite reumatoide e outras anormalidades, pelo menos por 10 a 15 anos em $90 \%$ dos procedimentos ${ }^{(1)}$. No entanto, pacientes submetidos à artroplastia total ou unicompartimental no joelho podem apresentar dor nessa articulação por presença de corpo livre, infecção, mau posicionamento dos componentes da prótese, soltura de um ou mais componentes, fratura periprotética ou, nos casos de prótese unicompartimental, lesão meniscal no compartimento não protético ${ }^{(2-4)}$.

Com o aumento do tempo e do número de pacientes em acompanhamento nos últimos anos, têm surgido novos métodos diagnósticos e modalidades terapêuticas; porém, grande número de casos com dor e disfunção de mobilidade traz dificuldade diagnóstica, exigindo extensa avaliação e experiência do cirurgião ortopédico para a melhora clínica e funcional dos pacientes. Wasilewski e Frank $1^{(2)}$ concluíram, em seu trabalho, que a artroscopia do joelho está indicada para pacientes com quadro de dor, déficit de mobilidade e sinovite, quando os exames de avaliação de rotina não são esclarecedores.

O propósito deste estudo foi investigar a causa da dor em pacientes sintomáticos após artroplastia no joelho por meio da realização da artroscopia, de maneira a avaliar o valor do procedimento como método diagnóstico e terapêutico entre os doentes não beneficiados por outros tipos de exames.

\section{CASUÍSTICA E MÉTODOS}

Este é um estudo prospectivo, no formato de série de casos, incluindo pacientes de um hospital universitário público. Dentre todos os pacientes submetidos à artroplastia para aplicação de próteses unicompartimentais, próteses totais ou artroplastia patelofemoral, foram selecionados para o estudo aqueles que permaneceram com sintomatologia dolorosa mesmo após o uso de analgésicos, anti-inflamatórios não-hormonais e fisioterapia (conforme prescrição individual) e cuja causa da dor não pôde ser esclarecida por exames de imagem, laboratoriais ou clínicos. Todos foram submetidos a artroscopia exploratória e terapêutica. A pesquisa foi aprovada pelo Comitê de Ética da instituição e os pacientes assinaram termos de consentimento livre e informado.
Entre setembro de 2001 e abril de 2007, 402 artroplastias foram realizadas por um mesmo cirurgião no serviço. Do total de 402 artroplastias, 114 pacientes receberam próteses unicompartimentais (PUC); 284 pacientes, prótese total do joelho (PTJ); e o restante (quatro pacientes) foi submetido à artroplastia patelofemoral (PPF).

Dentre os 402 operados, 17 pacientes permaneceram com dor, sem diagnóstico clínico, laboratorial ou por imagem que comprovasse a causa da sintomatologia dolorosa. Sete deles haviam recebido PUC (42,10\%); nove, PTJ (52,63\%); e um caso (5,27\%) havia sido submetido à PPF. Todos foram então submetidos à artroscopia indicada por causa da dor, em média oito meses após a artroplastia. A maioria era composta de mulheres (15 pacientes, $88,24 \%$ ) e a média de idade era de 65 anos.

A dor pré-artroscopia foi avaliada utilizando-se a escala de Lysholm $^{(5)}$, com resultados variando de 0 a 100 pontos. A melhora da dor tem resultado considerado ruim quando $<68$; regular, 69-76; bom, 77-90; e excelente, 91-100.

Foi adotado o nível de significância de 5\% $(0,05)$, para a aplicação dos testes estatísticos. Foi utilizado o programa SPSS (Statistical Package for Social Sciences), em sua versão 13.0, para a obtenção dos resultados. Foram aplicados o teste dos postos sinalizados de Wilcoxon e a análise de correlação de Spearman para avaliação estatística dos resultados de dor.

\section{RESULTADOS}

A avaliação dos pacientes pela escala de Lysholm demonstrou diferença significativa entre a medida pré e a pós-artroscopia (Tabela 1). O procedimento foi efetivo para o alívio da sintomatologia dolorosa em 14 dos pacientes $(82,35 \%)$, com resultado ruim em dois $(11,76 \%)$, que mantiveram o quadro álgico, e resultado regular em um caso $(5,88 \%)$; portanto, com resultado bom ou excelente nos demais casos avaliados (Tabela 2).

Tabela 1 - Avaliação de pacientes submetidos à artroplastia e sintomáticos pela escala de Lysholm antes e após artroscopia exploratória e terapêutica

\begin{tabular}{c|c|c|c|c|c|c|c}
\hline Variáveis & $n$ & Média & Desvio-padrão & Mínimo & Máximo & Mediana & $p$ \\
\hline Escore-PRÉ & 17 & 35,82 & 13,25 & 13,00 & 62,00 & 36,00 & \multirow{2}{*}{$<0,001^{*}$} \\
\hline Escore-PÓS & 17 & 86,18 & 15,56 & 47,00 & 100,00 & 94,00 & \\
\hline
\end{tabular}


Tabela 2 - Características dos 17 pacientes com artoplastia do joelho e sintomatologia dolorosa, submetidos à artroscopia

\begin{tabular}{|c|c|c|c|c|c|c|c|c|}
\hline No. & Sexo/ldade & Sintoma & Joelho & Artroplastia & Resultado & $\begin{array}{l}\text { Lysholm prélpós- } \\
\text { operatório }\end{array}$ & $\begin{array}{l}\text { Diagnóstico } \\
\text { artroscópico }\end{array}$ & Queixa atual \\
\hline 1 & $\mathrm{fem} / 61$ & Dor & D & PUC & Excelente & $18 / 93$ & "Ciclopes" & - \\
\hline 2 & $\operatorname{masc} / 54$ & Dor & $E$ & PUC & Excelente & $26 / 95$ & Sinovite anterior & - \\
\hline 3 & masc/55 & Dor & $D$ & PUC & Excelente & $55 / 100$ & "Ciclopes" & - \\
\hline 4 & fem/69 & Dor & D & PUC & Bom & $36 / 78$ & "Ciclopes" & Dor \\
\hline 5 & $\mathrm{fem} / 54$ & Dor & E & PUC & Regular & $19 / 73$ & Sinovite anterior & - \\
\hline 6 & fem $/ 56$ & Dor & $D$ & PUC & Excelente & $56 / 95$ & "Ciclopes" & - \\
\hline 7 & fem/59 & Dor & $D$ & PUC & Bom & $34 / 83$ & "Ciclopes" & - \\
\hline 8 & fem/67 & Dor & $\mathrm{D}$ & PPF & Excelente & $42 / 94$ & "Ciclopes" & - \\
\hline 9 & fem/68 & Dor & $D$ & PTJ & Excelente & $39 / 94$ & Sinovite anterior & - \\
\hline 10 & fem $/ 74$ & Dor & $\mathrm{D}$ & PTJ & Ruim & $36 / 47$ & Sinovite anterior & Dor \\
\hline 11 & fem/68 & Dor & $E$ & PTJ & Excelente & $39 / 97$ & "Ciclopes" & - \\
\hline 12 & $\mathrm{fem} / 73$ & Dor & D & PTJ & Excelente & $39 / 97$ & Sinovite anterior & - \\
\hline 13 & fem/77 & Dor & D & PTJ & Ruim & $13 / 53$ & "Ciclopes" & Dor \\
\hline 14 & fem/76 & Dor & $E$ & PTJ & Excelente & $30 / 97$ & "Ciclopes" & - \\
\hline 15 & fem/68 & Dor & $E$ & PTJ & Excelente & $62 / 97$ & "Ciclopes" & - \\
\hline 16 & fem/60 & Dor & $E$ & PTJ & Bom & $31 / 85$ & "Ciclopes" & - \\
\hline 17 & fem/63 & Dor & E & PTJ & Bom & $34 / 87$ & "Ciclopes" & - \\
\hline
\end{tabular}

A maioria dos pacientes (12 casos) apresentava como diagnóstico artroscópico um quadro de "ciclopes" (Fibrose intra-articular, Figura 1) e em cinco foi verificada sinovite anterior. Todos os pacientes foram submetidos a uma ressecção e liberação por via artroscópica do joelho (Figura 2).

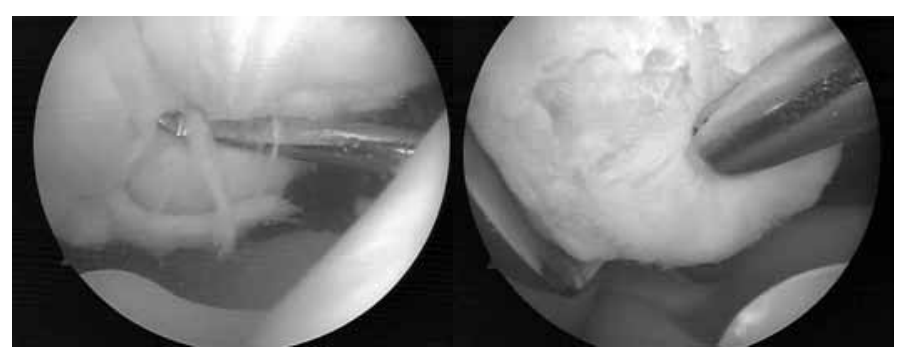

Figura 1 - "Ciclope" (fibrose), observado por artroscopia, entre os componentes da prótese, sendo ressecado com lâmina de shaver

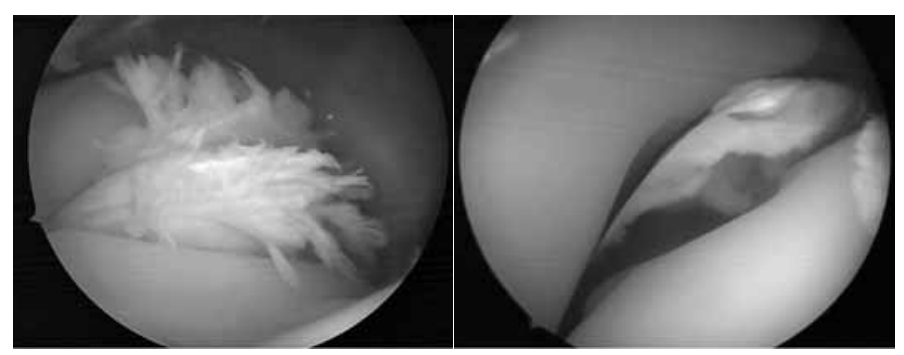

Figura 2 - "Ciclope" (fibrose), observado por artroscopia, entre os componentes da prótese e pós-ressecção

\section{DISCUSSÃO}

Há algum tempo a artroscopia vem sendo indicada como método diagnóstico e terapêutico para sintomas álgicos pós-artroplastia do joelho ${ }^{(6)}$. A artroscopia pode levar à indicação mais adequada de revisão de um componente ou de toda a prótese em casos de dúvida diagnóstica, sendo muito útil para a detecção de soltura de componentes protéticos, corpos livres intra-articulares, fraturas ou outras anormalidades ${ }^{(7-12)}$. Já se demonstrou, em trabalhos internacionais, que a artroscopia pode ser usada ainda como método de coleta de material por suspeita de infecção ou para evacuação de hematoma intra$\operatorname{articular}^{(6,7,13)}$, o que não foi necessário em nenhum caso neste estudo. No entanto, nenhum estudo prospectivo a esse respeito havia sido realizado com casuística brasileira até o momento e o presente trabalho serve para mostrar que em nosso meio o procedimento também é viável.

Diversos autores $^{(2,6,8,14-17)}$ demonstraram a importância da artroscopia como método diagnóstico e terapêutico para artrofibrose do joelho, levando a melhora significativa do quadro de dor e função do joelho acometido. Neste trabalho, não foi encontrada artrofibrose articular, mas apenas casos de fibrose localizada, que foi chamada de "ciclope"(2). Trata-se da presença de tecido fibroso interposto entre os componentes e não aderências articulares (Figuras 1 e 2). 
Como complicação do uso desse método, Diduch et $a l^{(3)}$ relatam $6 \%$ de pacientes com infecção articular após o procedimento, o que não foi demonstrado nos demais trabalhos avaliados ${ }^{(2,7,8,14,15,18-22)}$, em que a infecção não foi relatada ou com baixos índices de acometimento. Tampouco neste estudo foram observados casos de complicação do procedimento. Para Klinger et $a l^{(21)}$ é importante a antibioticoterapia preventiva no perioperatório para a minimização de complicações do método. Nos 17 pacientes deste estudo, apesar de não ter sido realizada a antibioticoterapia, não ocorreu infecção.

Diversos autores $^{(12,14,16,17,23,24)}$ têm reservado a cirurgia aberta somente para casos de revisão dos componentes protéticos ou em caso em que a retirada deles se faz essencial para o tratamento. Porém, existem autores ${ }^{(18)}$ que advogam o tratamento conservador, associando manipulação da articulação sob narcose. Entretanto, Lindenfeld et $a l^{(13)}$ descrevem que esses procedimentos podem levar à ruptura do tendão patelar, lesão intra-articular de tecidos ou síndrome de dor regional do joelho. Há, ainda, outras opções. Jerosch e Aldawoul$\mathrm{dy}^{(22)}$ defendem grandes benefícios da tríade: liberação artroscópica, controle da dor e fisioterapia intensa para pacientes com artrofibrose. O presente trabalho demons-

\section{REFERÊNCIAS}

1. Ranawat CS, Flynn WF Jr, Saddler S, Hansraj KK, Maynard MJ. Long-term results of total condylar knee arthoplasty. A 15-year survivorship study. Clin Orthop Relat Res. 1993;(286):94-102.

2. Wasilewski SA, Frankl U. Arthroscopy of the painful dysfunctional total knee replacement. Arthroscopy. 1989;5(4):294-7.

3. Diduch DR, Scuderi GR, Scott WN, Insall JN, Kelly MA. The efficacy of arthroscopy following total knee replacement. Arthroscopy. 1997;13(2):166-71.

4. Bocell JR, Thorpe CD, Tullos HS. Arthroscopic treatment of symptomatic total knee arthroplasty. Clin Orthop Relat Res. 1991;(271):125-34.

5. Lysholm J, Gillquist J. Evaluation of knee ligament surgery results with special emphasis on use of a scoring scale. Am J Sports Med. 1982;10(3):150-4.

6. Mintz L, Tsao AK, McCrae CR, Stulberg SD, Wright T. The arthroscopic evaluation and characteristics of severe polyethylene wear in total knee arthroplasty. Clin Orthop Relat Res. 1991;(273):215-22.

7. Havel PE, Giddings JC. Fracture of polyethylene tibial component in total knee arthroplasty diagnosed by arthroscopy. Orthopedics. 1994;17(4):357-8.

8. Wasilewski SA, FrankI U. Fracture of polyethylene of patellar component in total knee arthroplasty, diagnosed by arthroscopy. J Arthroplasty. 1989;4(Suppl):S19-22.

9. Johnson DR, Friedman RJ, McGinty JB, Mason JL, St Mary EW. The role of arthroscopy in the problem total knee replacement. Arthroscopy. 1990;6(1):30-2.

10. Hirsh DM, Sallis JG. Pain after total knee arthroplasty caused by soft tissue impingement. J Bone Joint Surg Br. 1989;71(4):591-2.

11. Bae DK, Lee HK, Cho JH. Arthroscopy of symptomatic total knee replacements. Arthroscopy. 1995;11(6):664-71.

12. Court C, Gauliard C, Nordin JY. Modalités techniques de l'arthrolyse arthroscopique après prothèse totale du genou. Rev Chir Orthop Reparatrice Appar Mot. 1999;85(4):404-10.

13. Lindenfeld TN, Wojtys EM, Husain A. Instructional course lectures, The American Academy of Orthopaedic Surgeons - Operative treatment of arthrofibrosis of the knee. J Bone Joint Surg Am. 1999;81:1772-84. Disponível em: http://www. ejbjs.org/cgi/content/extract/81/12/1772. Acessado em 2009 (18 fev.). trou bons resultados na melhora do quadro álgico dos pacientes com uso apenas da artroscopia diagnóstica e terapêutica para ressecção da fibrose localizada ou da sinóvia hipertrofiada.

Diversos trabalhos internacionais já falam a favor da cirurgia artroscópica para tratamento da dor pós-artroplastia ${ }^{(2,6,7,8,14,15,18-22)}$. O presente estudo é a primeira série de casos avaliados no Brasil e mostra que também em nosso meio a abordagem da dor pós-artroplastia do joelho pode ser realizada pela via artroscópica com vantagens clínicas, evidenciadas pela alta resolução e pela ausência de complicações do procedimento nesta série de casos.

\section{CONCLUSÃO}

A artroscopia do joelho pós-artroplastia, em pacientes com dor, sem um diagnóstico preestabelecido e já submetidos a tratamento conservador sem sucesso, foi benéfica, demonstrando melhora importante do quadro álgico conforme avaliado pela escala de Lysholm. Como causa da sintomatologia, foi verificado, na maioria dos pacientes, quadro de "ciclopes" (artrofibrose localizada) ou de sinovite, que pôde ser tratada no mesmo procedimento.

14. Arya RC. Arthroscopic arthrofibrolysis of the knee - Results of 28 cases. Arthroscopy. 2003;19(6 Suppl):80. (Paper 155).

15. Ayers DC, Dennis DA, Johanson NA, Pellegrini VD Jr. Instructional course lectures, The American Academy of Orthopaedic Surgeons - Common complications of total knee arthroplasty. J Bone Joint Surg Am. 1997;79:278-311. Disponível em: http://www.ejbjs.org/cgi/content/extract/79/2/278. Acessado em 2009 (18 fev.)

16. Kim J, Nelson CL, Lotke PA. Stiffness after total knee arthroplasty. Prevalence of the complication and outcomes of revision. J Bone Joint Surg Am. 2004;86(7):1479-84.

17. Tirveilliot F, Migaud H, Gougeon F, Laffargue P, Maynou C, Fontaine C. Traitement des raideurs sur prothèse totale du genou: indication des différents gestes mobilisateurs à propos de 62 cas. Rev Chir Orthop Reparatrice Appar Mot. 2003;89(1):27-34

18. Carro LP, Suarez GG. Intercondylar notch fibrous nodule after total knee replacement. Arthroscopy. 1999;15(1):103-5.

19. Krüger T, Reichel H, Decker T, Hein W. Arthroscopy after dysfunctional total knee arthroplasty: two cases with peg fracture of the polyethylene insert. Arthroscopy. 2000;16(8):E21.

20. Takahashi M, Miyamoto S, Nagano A. Arthroscopic treatment of soft-tissue impingement under the patella after total knee arthroplasty. Arthroscopy. 2002;18(4):E20.

21. Klinger HM, Baums MH, Spahn G, Ernstberger T. A study of effectiveness of knee arthroscopy after knee arthroplasty. Arthroscopy. 2005;21(6):731-8.

22. Jerosch J, Aldawouldy AM. Arthroscopic treatment of patients with moderate arthrofibrosis after total knee replacement. Knee Surg Sports Traumatol Arthrosc. 2007;15(1):71-7.

23. Christensen CP, Crawford JJ, Olin MD, Vail TP. Revision of the stiff total knee arthroplasty. J Arthroplasty. 2002;17(4):409-15.

24. Campbell ED Jr. Arthroscopy in total knee replacements. Arthroscopy. $1987 ; 3(1): 31-5$ 